\begin{tabular}{|l|c|c|c|c|}
\hline Cuadernos I. Geográfica & $20-21$ & pp. 23-45 & Logroño & 1994-95 \\
\hline
\end{tabular}

\title{
INFLUENCIA DE LA EXPOSICIÓN SOBRE LA PRODUCCIÓN DE SEDIMENTOS Y ESCORRENTÍAS EN AMBIENTES SEMIÁRIDOS
}

\begin{abstract}
ARTEMI CERDĀ 1
RESUMEN. Mediante experimentos con lluvia simulada se analiza la influencia de la exposición en la dinámica de la escorrentía y la erosión en una cuenca donde la vegetación se distribuye en un mosaico de macollas y zonas entre macollas de Stipa tenacissima. En estas últimas se distinguen tres tipos de superficies: Enlosados (con fragmentos de piedras), Encostrados (con costras) y Hierbas (Bracbypodium retusum).

La tasas de escorrentía y erosión son muy bajas en los suelos vegetados (Esparto y Hierbas) mientras que en las zonas entre matas (Encostrados y Enlosados) son más elevadas. La vegetación determina la generación de la escorrentía al controlar la distribución de las tasas de infiltración.

Tanto la escorrentía como la erosión están claramente influidas por la exposición. La ladera norte mantiene mayores tasas de infiltración final estable, la escorrentía es retrasada y escasa, y la pérdida de suelo es insignificante en comparación con la ladera sur.

Las relaciones entre la vegetación y la arroyada son negativas y no lineales, presentando umbrales muy marcados, lo que es debido a la distribución en manchas de la vegetación. La modelización y predicción de los procesos geomorfológicos en ambientes semiáridos es muy difícil debido a la elevada variabilidad espacial de sus factores, especialmente de la vegetación.
\end{abstract}

ABSTRACT. By means of experiments with simulated rainfall the influence of aspect on runoff and erosion dynamics is analysed in a basin where the vegetation is distributed within a mosaic of Stipa tenacissima L. shrubs and inter-shrubs. In the inter-shrubs areas three different types of areas exist: Stone pavement (with rock fragments),

1. Departament de Geografia. Universitat de València. 46080 Valencia 


\section{ARTEMI CERDÀ}

Crusted surfaces (with surface crust) and vegetation covered areas (Brachypodium retusum).

The erosion and runoff rates are very low beneath the vegetation (Esparto tussocks and Brachypodium retusum grass), while in the intershrubs areas (Crusted and Stone pavement) rates are higher. The vegetation controls runoff generation through its effect on the infiltration rates.

Both runoff and erosion processes are influenced by aspect. The north-facing slopes have bigher infiltration rates, and runoff is attenuated and reduced. The soil losses are neglegible in compararison to the south-facing slope.

Vegetation cover is inversely and non-linearly related to the volume of overland flow, and the relationship exhibit a threshold due to the patch distribution of the vegetation. Modelling and predictioning the geomorphological processes under semiarid conditions is very complicated due to the high spatial variability of his factors, specially the vegetation.

Palabras clave: Exposición, Erosión, Escorrentía, Semiárido, Lluvia simulada Key words: Aspect, Erosion, Runoff, Semiarid, Rainfall simulation

\section{Introducción}

La importancia de la exposición en el control de los procesos geomorfológicos ha sido reconocida por diversos autores (GREGORY y WALLING, 1973). La radiación recibida en laderas de exposición contrastada hace que su régimen hídrico sea muy distinto (GONZÁLEZ HIDALGO, 1992). Por ello, la exposición contrastada genera diferencias en la cubierta vegetal y en la composición florística (MARGALEF, 1986). En latitudes medias y altas, estas diferencias son debidas a las mayores temperaturas y tasas de evaporación en las laderas sur, lo que genera en ambientes semiáridos un mayor stress hídrico y con ello menores cubiertas vegetales (GONZÁLEZ HIDALGO, 1994).

Además, las distintas condiciones climáticas debidas a la exposición explican la existencia de distintos procesos edáficos y tipos de suelo. Textura, estructura, contenido en materia orgánica, sales, etc., son parámetros diferenciadores de los suelos situados en las laderas norte y sur. Así los principales procesos geomorfológicos tienen tasas de actuación diferentes en laderas situadas en la misma latitud pero con exposición contrastada, incluso los procesos dominantes son distintos, lo que da lugar a geoformas dispares (CHURCHILL, 1981; CHURCHILL, 1982; KIRKBY et al., 1986; WEAVER, 1991). 
INFLUENCIA DE LA EXPOSICIÓN SOBRE LA PRODUCCIÓN DE SEDIMENTOS Y ESCORRENTÍAS

Aunque se han realizado estudios previos, es poco conocida la influencia de la exposición sobre la escorrentía y la erosión. Además no existen muchos trabajos en los que se estudie el tema desde el punto de vista de la interacción entre la vegetación y la exposición, aproximación que parece básica para entender el funcionamiento de los sistemas geomórficos semiáridos, donde la vegetación condiciona la arroyada superficial (THORNES, 1976; COOKE et al., 1993; ABRAHAMS y PARSONS, 1994).

El objetivo del trabajo es determinar la influencia de la exposición sobre la generación de escorrentía y movilización de sedimentos, haciéndose especial hincapié en la distribución espacial de ambos procesos en un ambiente semiárido. Para ello se estudia el control que ejerce la vegetación en dos laderas de exposición contrastada sobre la generación de la arroyada a partir de experimentos con lluvia simulada. Los chaparrones simulados son idénticos en intensidad y duración con el fin de eliminar la variabilidad introducida por la lluvia natural y reducir los costes y el tiempo de toma de datos.

\section{2. Área de estudio}

Al norte de la provincia de Alicante, entre los términos municipales de Benidorm y Finestrat, se ha seleccionado un valle con laderas de exposición contrastada. Esta zona está situada en la vertiente sur de la Serra d'Aitana, dentro del Sistema Prebético. El valle se ha desarrollado totalmente sobre calizas cretácicas, si bien existen en la base de las laderas depósitos cuaternarios en los que dominan los cantos angulosos (Fig. 1).

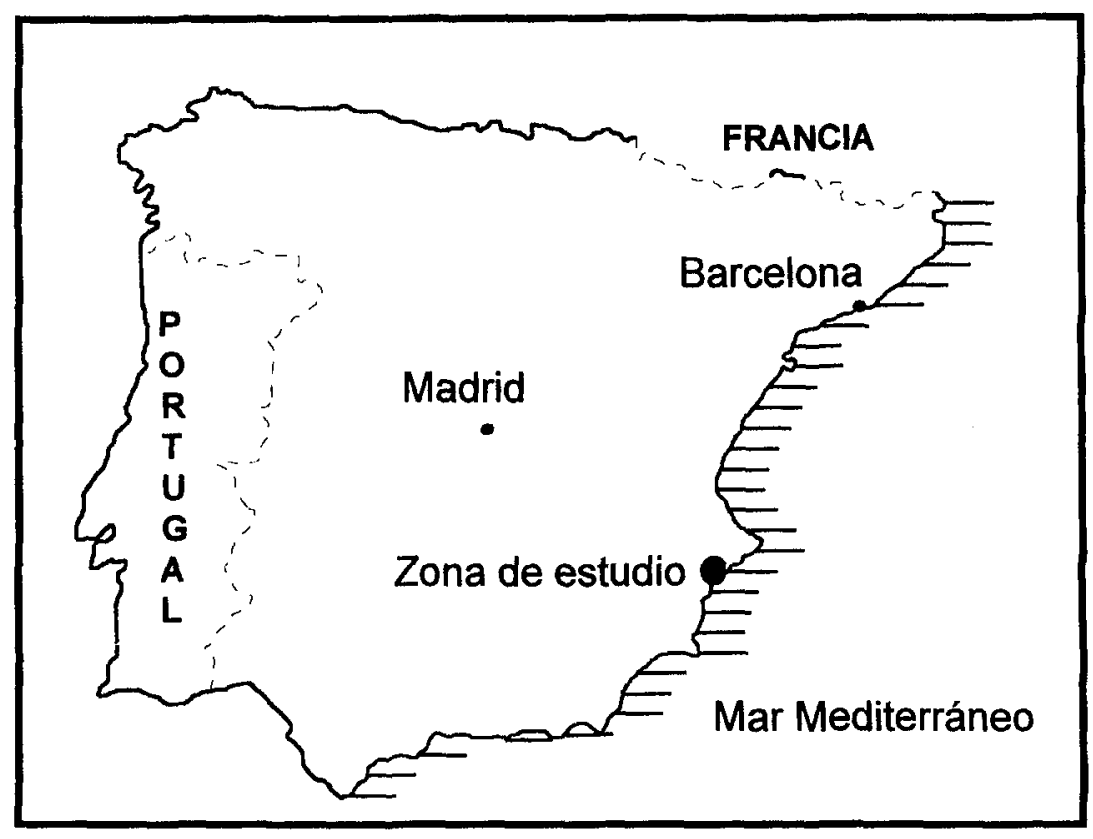

Figura 1. Localización de la zona de estudio 
Las dos laderas estudiadas presentan un perfil convexo, existiendo en la parte baja campos de cultivo de algarrobo, en la actualidad abandonados y colonizados por Pinus halepensis de forma natural (Fig. 2). Parece clara la relación entre la forma de las laderas y la construcción de los campos de cultivo, los cuales zaparon la base de las laderas y con ello modificaron la forma cóncava original. Hay evidencias de campos de cultivo muy antiguos en algunas laderas, pero parece que no proliferaron demasiado. Esto último es debido a la aridez de la zona, con $350 \mathrm{~mm}$ anuales de precipitación media, alta irregularidad interanual, elevada evapotranspiración, prolongados periodos de sequía, etc. Por ello, el cultivo se ha centrado en el fondo de valle, donde al contrario que en las laderas se produce una acumulación de sedimentos y nutrientes, y lo que es más importante, de agua. Las temperaturas mensuales medias oscilan entre $22,18{ }^{\circ} \mathrm{C} \mathrm{y}$ $12,85^{\circ} \mathrm{C}$. La influencia del mar hace que se produzca una suavización de $1-2{ }^{\circ} \mathrm{C}$. Los días de heladas son insignificantes: 1,4 días por año.

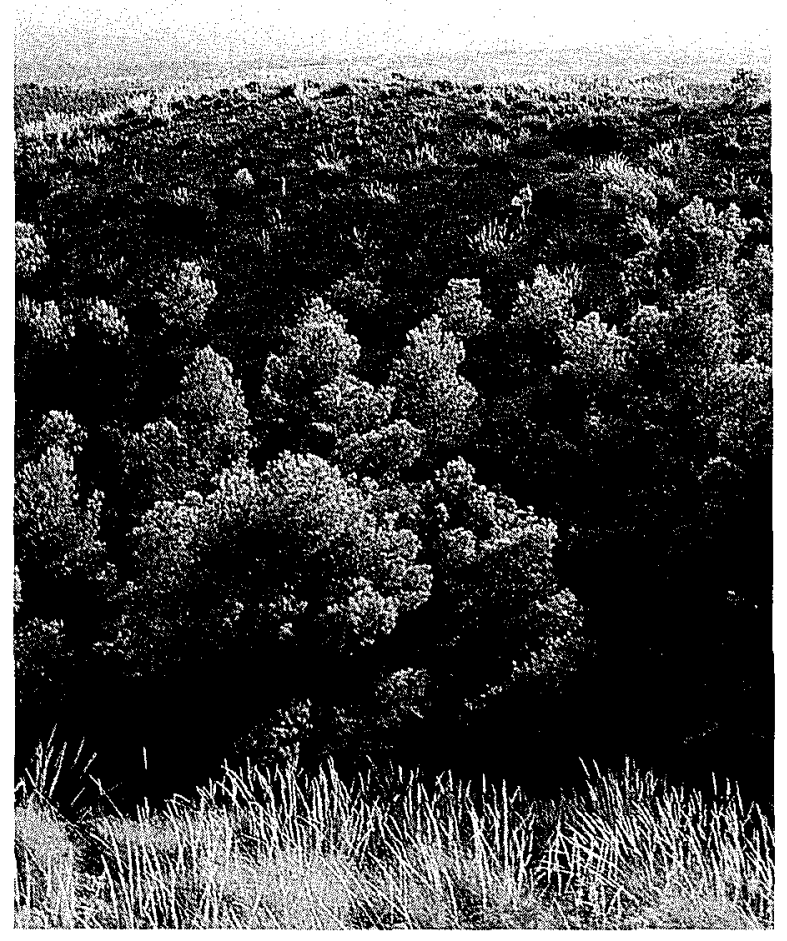

Figura 2a. Vista de la zona de estudio. Ladera norte 


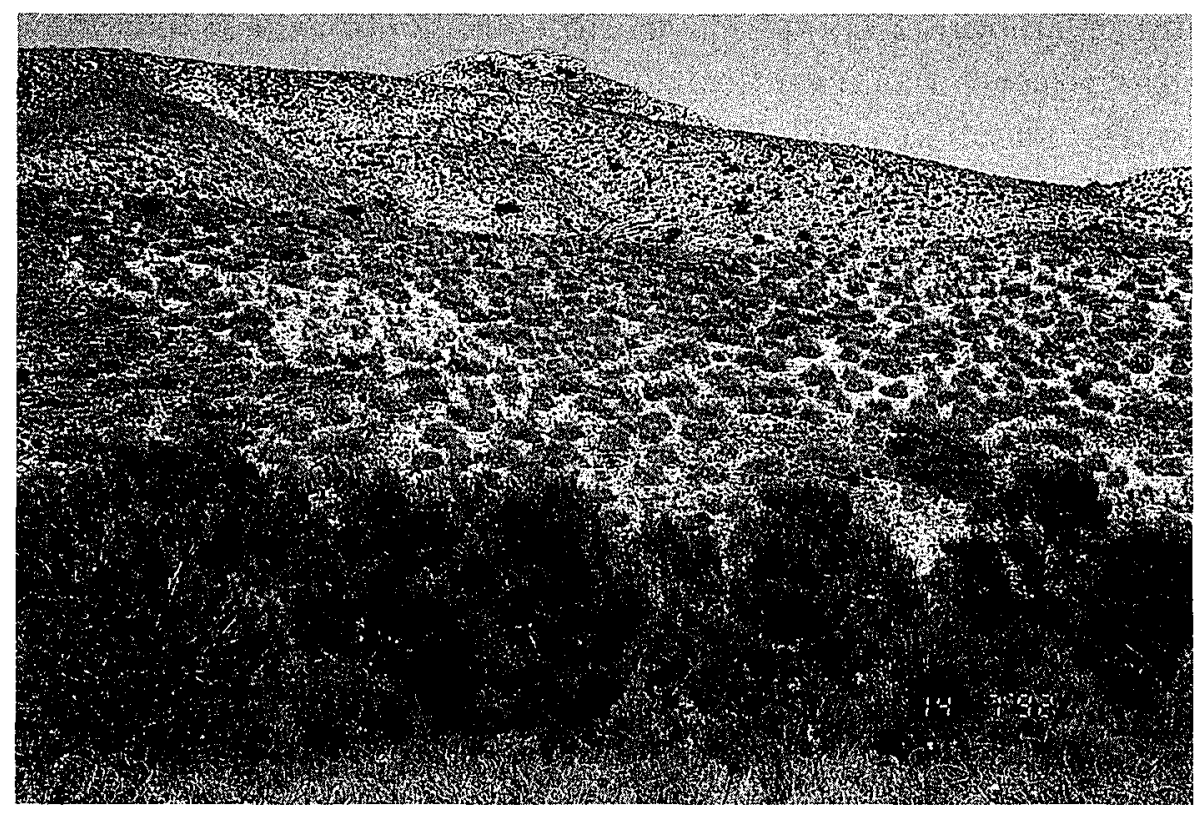

Figura 2b. Vista de la zona de estudio. Ladera sur

La vegetación en la ladera sur está compuesta básicamente por Stipa tenacissima, aunque otras especies como Brachypodium retusum y Fumana ericoides también aparecen de forma reiterada. Especies como Pinus balepensis, Pistacia lentiscus y Ceratonia siliqua sólo existen en la parte baja de la ladera y campos de cultivo, mientras que Rosmarinus officinalis, Thymus vulgaris y Rhamnus lyciodes aparecen esporádicamente. En la laderas norte la diversidad es mayor, despareciendo la preponderancia de Stipa tenacissima. Otras especies como Pistacia lentiscus, Brachypodium retusum y Pinus halepensis, junto a Rosmarinus officinalis, Thymus vulgaris, Erica multiflora, Globularia alypum, Mirtus sp. y Fumana coexisten junto a líquenes y musgos, los cuales aprovechan el favorable régimen hídrico de la umbría.

Los suelos, de elevada pedregosidad superficial, están pobremente desarrollados y presentan perfiles con profundidades de tan sólo $10 \mathrm{~cm}$. Los cuatro perfiles muestreados a lo largo de la ladera sur y los cinco de la norte, han permitido clasificar los suelos como Leptosoles líticos. A pesar de su pobre desarrollo, los suelos presentan una buena estructura (CALVO et al., 1994), debido a la importante actividad biológica, directamente relacionada con la población de lombrices que determinan la calidad de la agregación del suelo (BOIX et al., 1994).

Para los cuatro suelos muestreados en la ladera sur el $\mathrm{pH}$ medio es 8,27 en el horizonte A y 8,23 en el AC. La conductividad eléctrica varía entre 0,24 y $0,37 \mu \mathrm{S}$ y el contenido en materia orgánica (2,02-6,43\%) es elevado en relación a las reducidas precipitaciones y la exposición sur de la ladera. La textura es franco- 
arcillosa y presenta reducidas variaciones en profundidad. Los suelos están satu-

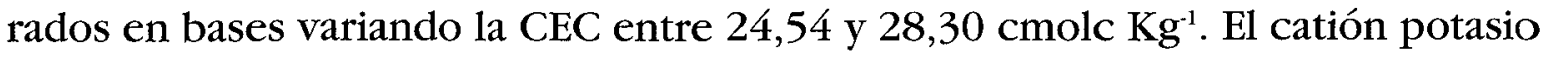
presenta valores de intercambio entre 0,37 y $0,78 \mathrm{cmolc}_{\mathrm{Kg}}{ }^{-1}$, y el calcio entre 19,22 y $27,48 \mathrm{cmolc} \mathrm{Kg}^{-1}$. El sodio presenta mayores oscilaciones (de 0,03 a 0,80 cmolc $\mathrm{Kg}^{-1}$ ), mientras que el magnesio varía entre 1,62 y 2,74 cmolc $\mathrm{Kg}^{-1}$.

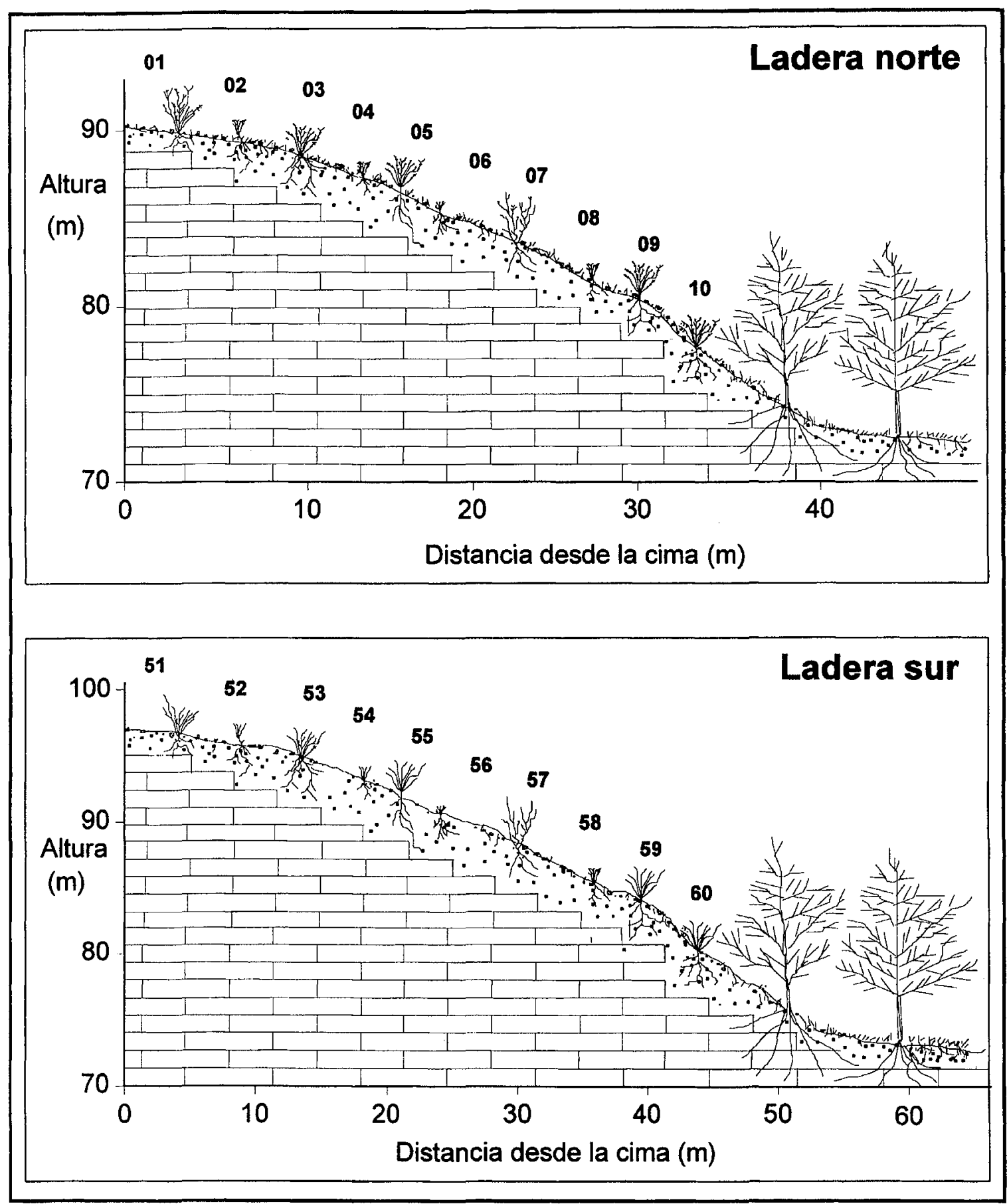

Figura 3. Croquis de las laderas estudiadas en Benidorm. Los números corresponden a la localización de las parcelas 
Para los cinco suelos muestreados en la ladera norte el $\mathrm{pH}$ es ligeramente superior a 8 , la conductividad eléctrica varía entre 0,18 y $0,33 \mu \mathrm{S}$ y el contenido en materia orgánica es elevado $(3,60-6,35 \%)$. También en esta ladera la textura es franco-arcillosa y presenta reducidos cambios en profundidad. Los suelos están saturados en bases oscilando la CEC entre 15,88 y $26,8 \mathrm{cmolc} \mathrm{Kg}^{-1}$ en el horizonte superficial y entre 20,94 y $31,55 \mathrm{cmolc} \mathrm{Kg}^{-1}$ en profundidad. El catión potasio presenta valores de intercambio entre 0,32 y 0,70 cmolc $\mathrm{Kg}^{-1}$, el calcio

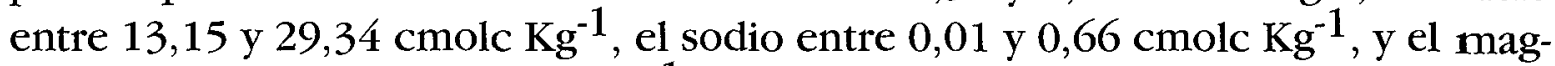
nesio entre 0,72 y $2,74 \mathrm{cmolc} \mathrm{Kg}^{-1}$ (SORIANO et al., 1995).

\section{Material y métodos}

Las mediciones de la pérdida de suelo y agua se han realizado mediante experimentos con lluvia simulada con el fin de eliminar la heterogeneidad de la lluvia natural y generar una amplia base de datos en un reducido periodo de tiempo. El simulador de lluvia utilizado ha sido del tipo pulverizador y la metodología aplicada en el campo ha sido la misma que en CERDÀ (1993), con una intensidad de la lluvia de $55 \mathrm{~mm} \mathrm{~h}^{-1}$ mantenida durante 60 minutos. Durante los experimentos se tomaron muestras de escorrentía cada minuto y de sedimentos entre 2 y 4 veces. También se determinó visualmente el inicio del encharcamiento ( 40 $\%$ de la superficie) y el de la escorrentía. Los chaparrones simulados tienen periodos de recurrencia de unos diez años en la zona de estudio (ELÍAS y RUIZ, 1979).

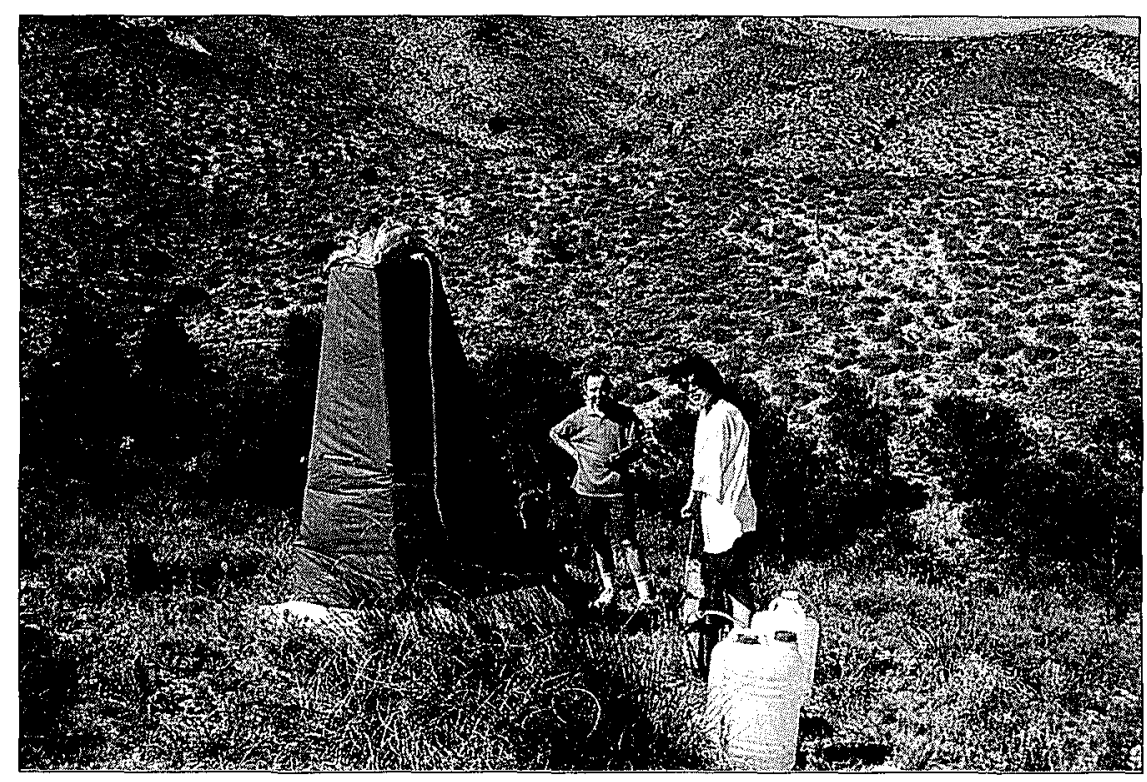

Figura 4. Simulador de lluvia 
ARTEMI CERDÀ

Los experimentos se realizaron en el verano de 1992, por lo que la humedad del suelo era muy baja. Las parcelas son de tamaño reducido $\left(0,24 \mathrm{~m}^{2}\right)$ ya que la distribución de la vegetación -especialmente en la ladera sur- está estructurada en matas y pasillos entre-matas. Sólo parcelas menores de $1 \mathrm{~m}$ de diámetro pueden abarcar superficies homogéneas.

En ambas laderas los regueros son inexistentes y las grietas son poco abundantes y de reducido tamaño $(<1 \mathrm{~mm})$. La rocosidad sólo aparece en la parte alta de las laderas, especiamente en la sur, aumentando ladera abajo la profundidad del suelo. Los líquenes y musgos, aunque existen, son poco abundantes. En las partes bajas de las laderas las zapas basales han sido practicadas con el fin de construir los campos de cultivo.

En la selección de las parcelas fue prioritario que estuvieran representados todos los tipos de superficies existentes (tabla 1). Las cuatro superficies tipo seleccionadas han sido: Matorral (esparto), Hierbas (Brachypodium retusum) acompañado normalmente de arbustos de bajo porte como (Thymus vulgaris o Fumana ericoides), Enlosados (piedras) y Encostrados (costra). Un ejemplo de estos tipos de superficie se presentan en las figuras $5 \mathrm{a}, 5 \mathrm{~b}, 5 \mathrm{c}$ y $5 \mathrm{~d}$.

Tabla 1. Vegetación y tipo de superficie existente en cada parcela. Cubierta vegetal insignificante (isg)

\begin{tabular}{|ccc|}
\hline NORTE & Vegetación & Superficie \\
\hline BE01 & Stipa tenacissima & Matorral \\
BE02 & Brachypodium retusum, Fumana ericoides & Hierbas \\
BE03 & Brachypodium retusum, Thymus vulgaris & Hierbas \\
BE04 & Thymus vulgaris, Fumana ericoides & Hierbas \\
BE05 & Brachypodium retusum, Fumana ericoides & Hierbas \\
BE06 & Brachypodium retusum & Piedras \\
BE07 & Brachypodium retusum, Fumana ericoides & Hierbas \\
BE08 & Brachypodium retusum, Erica multiflora & Piedras \\
BE09 & Globularia alypum, Fumana ericoides & Hierbas \\
BE10 & Rosmarinus officinalis & Matorral \\
\hline SUR & Vegetación & Superficie \\
\hline BE51 & Fumana ericoides (isg) & Costra \\
BE52 & Stipa tenacissima, Thymus vulgaris & Matorral \\
\hline
\end{tabular}


INFLUENCIA DE LA EXPOSICIÓN SOBRE LA PRODUCCIÓN DE SEDIMENTOS Y ESCORRENTÍAS

\begin{tabular}{|ccc|}
\hline BE53 & Brachypodium retusum, Fumana ericoides & Hierbas \\
BE54 & (isg) & Costra \\
BE55 & Stipa tenacissima & Matorral \\
BE56 & (isg) & Piedras \\
BE57 & Brachypodium retusum & Hierbas \\
BE58 & (isg) & Costra \\
BE59 & (isg) & Piedras \\
BE60 & Stipa tenacissima & Matorral \\
\hline
\end{tabular}

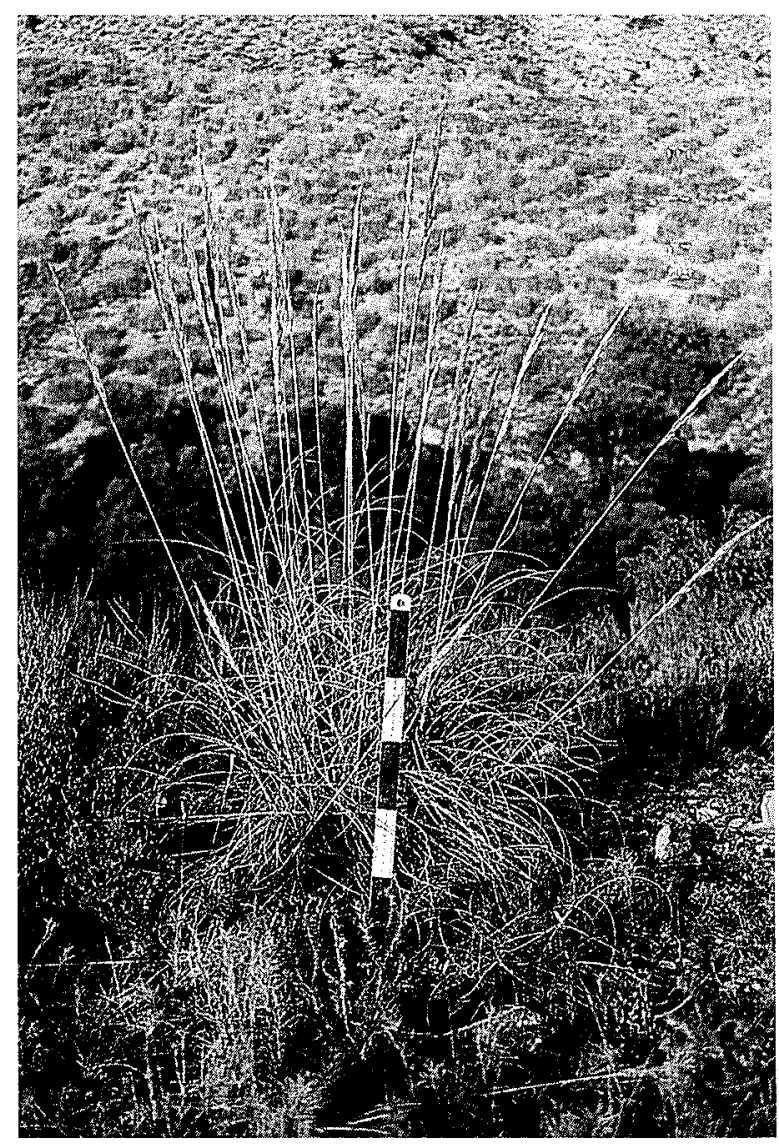

Figura 5a. Superficie dominada por Stipa tenacissima (BEO1). Matorral de esparto 


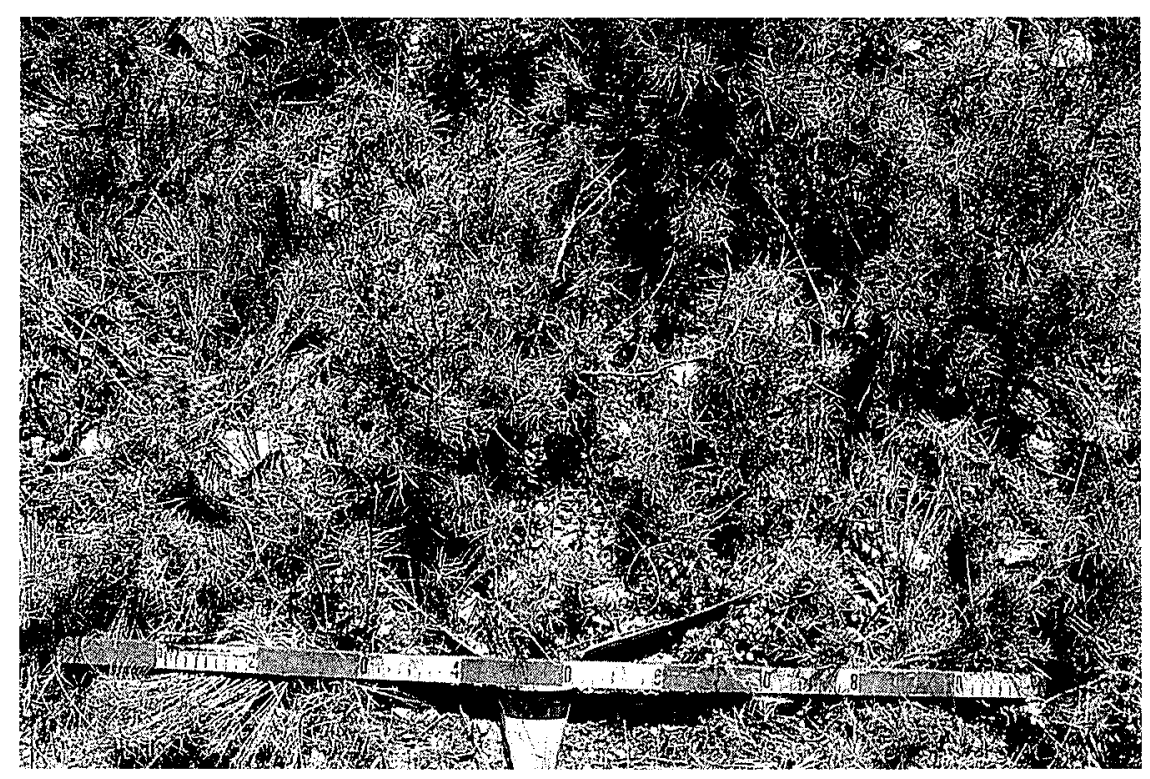

Figura 5b. Superficie dominada por Brachypodium retusum (BE06). Hierbas

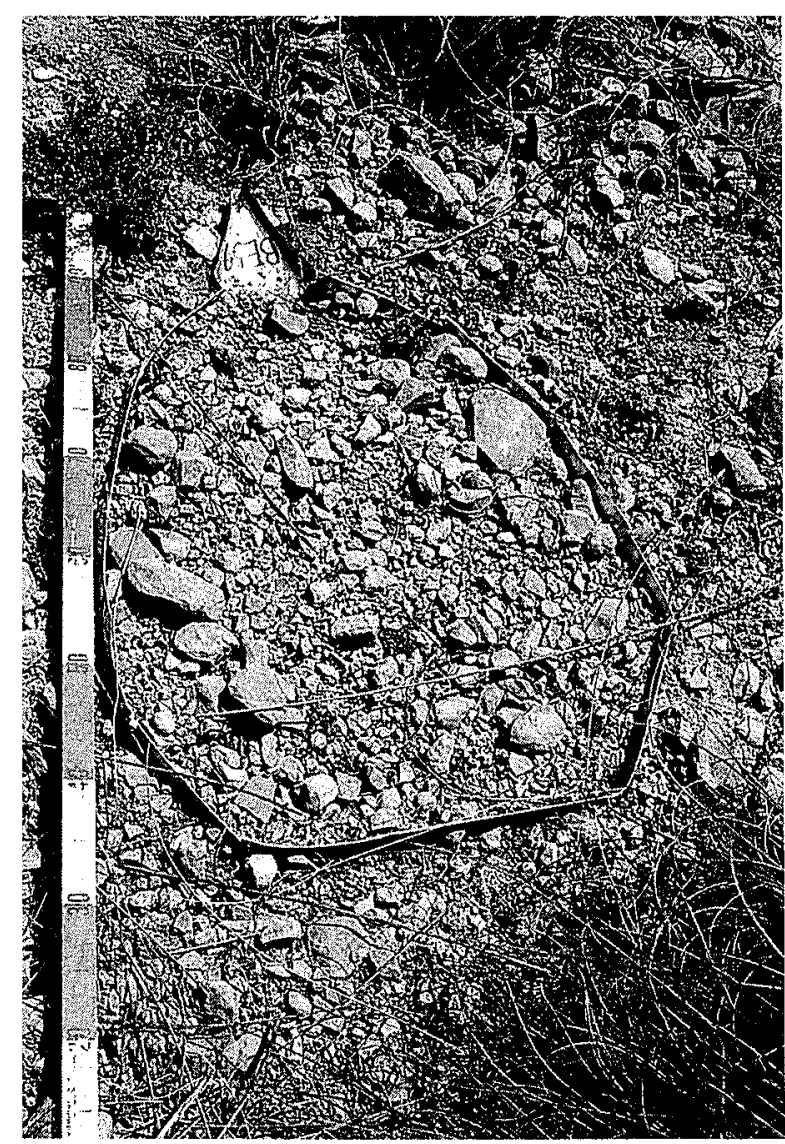

Figura 5c. Superficie dominada por las piedras (BE59). Enlosado 


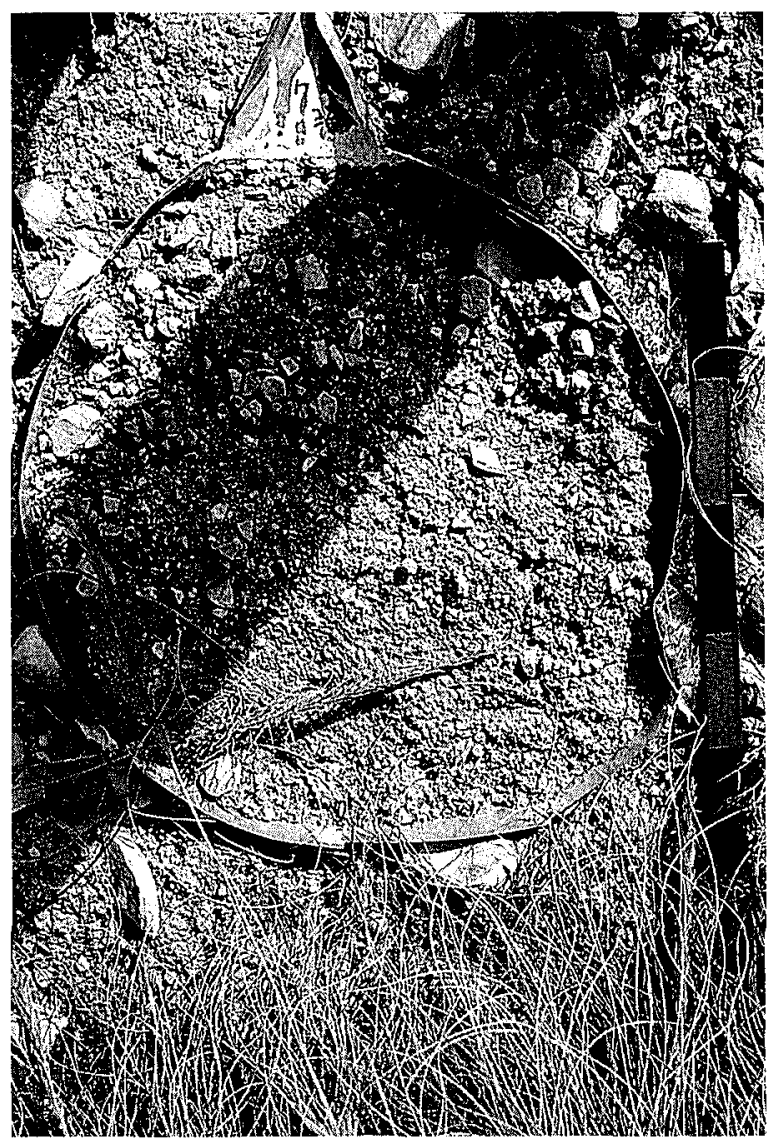

Figura 5d. Surpeficie dominada por las costras (BE54). Encostrado

\section{Resultados}

\subsection{Características de los suelos}

La pendiente de las parcelas varía entre $7^{\circ}$ y $28^{\circ}$. Esto se debe tanto a la forma de la ladera, como a la topografía generada por las macollas de esparto. El perfil de la ladera es convexo, por lo que los suelos de la parte alta tienen menores pendientes que los localizados en otras zonas de la ladera. La pendiente media es mayor en la ladera sur $\left(22^{\circ}\right)$ que en la ladera norte $\left(17^{\circ}\right)$, pero la forma de la ladera es semejante, convexa.

Las superficies vegetadas alcanzan cubiertas de hasta el $100 \%$, aunque en todas las parcelas siempre existen claros alrededor de las matas, de ahí que los valores oscilen entre el 50 y el 90 \% (tabla 2). La altura máxima de la vegetación está directamente relacionada con las especies dominantes en cada parcela, así la Stipa tenacissima presenta valores entre 30 y $50 \mathrm{~cm}$, mientras que Brachypodium retusum alcanza tan sólo $20 \mathrm{~cm}$ y los matorrales de bajo porte (Thymus vulgaris o Fumana ericoides) $30 \mathrm{~cm}$. En los suelos no vegetados, Enlosados o Encostrados, además de tener una cubierta vegetal insignificante, la 
ARTEMI CERDÀ

altura de ésta es reducida y en todo caso responde a arbustos esqueléticos o herbáceas aisladas. La mayor cubierta vegetal en la ladera norte ( $43 \%$ frente a $38 \%$ en la sur) está directamente relacionada con la mayor altura $(23 \mathrm{~cm}$ frente a 14 cm en la sur) y densidad de la vegetación.

Además de la mayor cubierta vegetal de la ladera norte, ésta es más homogénea en su distribución. En la ladera sur, la distribución de la vegetación en zonas de matas y zonas pasillo entre matas es la característica más destacable. Esto también está relacionado con la distribución de los líquenes y musgos. En la ladera norte tanto líquenes (10\%) como musgos (19\%) aparecen en todos los ambientes. En la ladera sur ( 2 y $6 \%$ respectivamente), tan sólo se pueden encontrar en las zonas protegidas por las macollas de esparto. Las zonas desnudas entre-matas están restringuidas a costras de origen físico y/o químico y a una cubierta de fragmentos de roca que se localiza sobre la costra y no dentro de ésta.

Obviamente la vegetación determina la distribución espacial de la hojarasca, la cual es muy elevada en el esparto debido a que no se explota, ya que se caracteriza por tener muy bajas tasas de descomposición. También es elevada la cubierta de hojarasca donde existe Brachypodium retusum. Los Enlosados y los Encostrados tienen insignificantes cubiertas de hojarasca.

La ladera sur tiene valores de hojarasca ligeramente menores a la ladera norte (15 y $17 \%$ respectivamente). Esta reducida diferencia es debida a que la hojarasca del esparto tiene muy bajos índices de descomposición, y a que es más abundante en la ladera sur. En la ladera norte, aunque la producción de hojarasca debe ser mucho mayor se caracteriza por una más rápida descomposición.

Tabla 2. Caracteristicas de las superficies estudiadas.

\begin{tabular}{|ccccccccc|}
\hline PARCELAS & $\begin{array}{c}\text { Pen } \\
\left({ }^{\circ}\right)\end{array}$ & $\begin{array}{c}\text { Pla } \\
(\%)\end{array}$ & $\begin{array}{c}\text { Ave } \\
(\%)\end{array}$ & $\begin{array}{c}\text { Liq } \\
(\%)\end{array}$ & $\begin{array}{c}\text { Mus } \\
(\%)\end{array}$ & $\begin{array}{c}\text { Hoj } \\
(\%)\end{array}$ & $\begin{array}{c}\text { Ped } \\
(\%)\end{array}$ & $\begin{array}{c}\text { MO } \\
(\%)\end{array}$ \\
\hline NORTE & & & & & & & & \\
BE01 & 7 & 90 & 50 & 2 & 6 & 50 & 30 & 5,37 \\
BE02 & 7 & 50 & 16 & 2 & 8 & 10 & 80 & 4,20 \\
BE03 & 12 & 50 & 30 & 5 & 50 & 15 & 70 & 5,64 \\
BE04 & 20 & 45 & 18 & 15 & 12 & 12 & 90 & 4,83 \\
BE05 & 21 & 80 & 14 & 4 & 17 & 23 & 50 & 6,35 \\
BE06 & 20 & 40 & 20 & 20 & 24 & 10 & 40 & 5,40 \\
BE07 & 15 & 5 & 6 & 15 & 20 & 13 & 20 & 4,47 \\
BE08 & 20 & 5 & 8 & 2 & 2 & 5 & 60 & 3,60 \\
\hline
\end{tabular}


INFLUENCIA DE LA EXPOSICIÓN SOBRE LA PRODUCCIÓN DE SEDIMENTOS Y ESCORRENT'IAS

\begin{tabular}{|ccccccccc|}
\hline PARCELAS & Pen & Pla & Ave & Liq & Mus & Hoj & Ped & MO \\
\hline BE09 & 20 & 20 & 25 & 30 & 40 & 10 & 40 & 4,42 \\
BE10 & 15 & 45 & 45 & 5 & 12 & 24 & 70 & 4,19 \\
Media & 15,7 & 43 & 23,2 & 10 & 19,1 & 17,2 & 55 & 4,85 \\
SUR & & & & & & & & \\
BE51 & 15 & 2 & 3 & 0 & 0 & 1 & 10 & 2,02 \\
BE52 & 18 & 50 & 30 & 1 & 3 & 12 & 65 & 6,43 \\
BE53 & 23 & 60 & 14 & 0 & 5 & 10 & 60 & 5,12 \\
BE54 & 20 & 1 & 1 & 0 & 0 & 1 & 15 & 2,94 \\
BE55 & 25 & 80 & 29 & 7 & 15 & 32 & 70 & 2,69 \\
BE56 & 24 & 0 & 0 & 5 & 12 & 0 & 90 & 3,62 \\
BE57 & 28 & 90 & 16 & 0 & 0 & 50 & 70 & 4,29 \\
BE58 & 20 & 2 & 1 & 0 & 0 & 0 & 15 & 3,49 \\
BE59 & 20 & 1 & 0 & 0 & 0 & 1 & 95 & 2,69 \\
BE60 & 25 & 90 & 45 & 10 & 20 & 40 & 30 & 3,77 \\
Media & 21,8 & 37,6 & 13,9 & 2,3 & 5,5 & 14,7 & 52 & 3,71 \\
\hline
\end{tabular}

Pen, Pendiente; Pla, Plantas; Ave, Altura máxima de la vegetación; Liq, Líquenes; Mus, Musgos; Hoj, Hojarasca; Ped, Pedregosidad y MO, Materia orgánica $(0-2 \mathrm{~cm}$ de profundidad)

La pedregosidad superficial es muy elevada en ambas laderas. Si la ladera sur no tiene valores mayores es porque existen suelos desprotegidos de piedras, los Encostrados, que en caso de la ladera norte son insignificantes. La cubierta de fragmentos de rocas también es muy elevada en el resto de las superficies, Hierbas y Matorral, pero no en el caso de los Encostrados. Esto significa que los suelos vegetados, además de la protección de la vegetación cuentan con una importante cubierta de piedras.

La diferencia en la distribución de la pedregosidad entre la ladera sur -más heterogénea- que la ladera norte -más homogénea- se debe a la existencia en la primera de zonas de acumulación de cantos y bloques. Ésta presenta formas alargadas en la dirección de la pendiente, lo que sugiere la existencia de "coladas" o "canchales" que se desplazan hacia la base de la ladera. Será necesario un estudio más detallado para confirmar o desmentir la existencia de estos procesos.

La materia orgánica es mayor por término medio en la ladera norte $(4,85 \%)$ que en la sur (3,71\%). A pesar de ello, en la ladera sur también existen zonas como las cubiertas por esparto, donde la materia orgánica es más abundante (tabla 2). 


\section{ARTEMI CERDÀ}

\subsection{Respuesta bidrológica}

La humedad del suelo durante el verano es muy baja, 3,3\% en superficie y $4,9 \%$ a cinco centímetros de profundidad. Paradójicamente los suelos de la ladera norte tienen menor humedad gravimétrica que los de la ladera sur: 3,54 frente a $3,05 \%$ en superficie $(0-2 \mathrm{~cm})$ y 6,3 frente a $3,53 \%$ en profundidad $(4-6 \mathrm{~cm})$, aunque las diferencias son poco apreciables respecto a la capacidad de retención hídrica de los suelos.

La respuesta de los suelos ante la lluvia se caracteriza por encharcamientos rápidos en los suelos Encostrados (1'55") y Enlosados (1'54") que dominan la zona entre matas de la ladera sur (tabla 3). En el Matorral, con esparto en ambas laderas y romero sólo en la norte, el encharcamiento es más retrasado (5'40"), mientras que en las superficies vegetadas con hierbas el encharcamiento bien es insignificante, no existe, o bien es muy retrasado.

La escorrentía se produce unos minutos después del encharcamiento y se mantienen las diferencias entre los suelos vegetados y los desnudos. En ocasiones, la escorrentía alcanza el colector después de 15 minutos, y en estos casos sus caudales son menores de $2 \mathrm{~mm} \mathrm{~h}^{-1}$. La diferencias entre la ladera sur y la norte son evidentes tanto en el encharcamiento como en el inicio de la escorrentía. Así, en término medio, encharcamiento y escorrentía, necesitan para producirse el doble de tiempo en la ladera norte (6' 62" y 9' 86") que en la ladera sur (2' 50" y 4'36" respectivamente).

Las tasas medias de escorrentía (Em) son bajas en ambas laderas lo que da lugar a hidrogramas con bajos caudales (Fig. 6) o incluso sin escorrentía. Hay una clara influencia de la distribución de la cubierta vegetal sobre la generación de la escorrentía, la cual es muy evidente en la ladera sur por el contraste entre las manchas de vegetación y las zonas pasillo. La precipitación total en cada experimento fue de $55 \mathrm{~mm}$ durante una hora, a pesar de lo cual los valores de $\mathrm{Em}$ han sido insignificantes para los suelos vegetados. En el Matorral y en los Hierbas (Brachypodium retusum), los valores medios de $\mathrm{Em}$ no alcanzan los $2 \mathrm{~mm}$, hasta el punto que no ha existido en algunos casos (BE53 y BE57). Hay que decir que en el caso del esparto, la escorrentía procede de las zonas no vegetadas que rodean la macolla, pero que está dentro de la parcela, si bien el suelo que corresponde a la planta es capaz de almacenar toda el agua precipitada. Los Encostrados tienen las mayores escorrentías (hasta $22 \mathrm{~mm}$ ), mientras que en los Enlosados, éstas son ligeramente inferiores (hasta $16 \mathrm{~mm}$ ).

Las diferencias entre ambas laderas son evidentes en valores medios: en la norte $\mathrm{Em}$ no alcanza los $2 \mathrm{~mm}$, mientras que en la sur supera los $9 \mathrm{~mm}$. Las anteriores diferencias son ratificadas por los coeficientes de escorrentía: $4 \%$ y $16 \%$ respectivamente. Esto se debe a que las Hierbas y el Matorral tienen coeficientes de escorrentía muy reducidos 0-1,07\% y 0-4,65 \%, mientras que los Enlosados 
INFLUENCIA DE LA EXPOSICIÓN SOBRE LA PRODUCCIÓN DE SEDIMENTOS Y ESCORRENTÍAS

(16,39-40,68 \%) y los Encostrados (6,15-30,82\%) producen las mayores pérdidas de agua. La mayor cantidad de superficies del primer tipo en la ladera norte, especialmente Hierbas, y de las segundas en la ladera sur, explican las diferencias.

La tasa de infiltración final estable es mayor en los suelos vegetados ( $54 \mathrm{y}$ $52 \mathrm{~mm} \mathrm{~h}^{-1}$ en las Hierbas y el Esparto) que en los no vegetados (44 y $35 \mathrm{~mm} \mathrm{~h}^{-1}$ en los Enlosados y Encostrados, respectivamente). Por ello, las tasas de infiltración final estable son mayores en la ladera norte $\left(52,4 \mathrm{~mm} \mathrm{~h}^{-1}\right)$ que en la ladera sur $\left(43,3 \mathrm{~mm} \mathrm{~h}^{-1}\right)$, siempre en término medio.

Tabla 3. Cambios en/de la superficie de los suelos ante la lluvia simulada (55 $\left.\mathrm{mm} \mathrm{h}^{\mathbf{1}}\right)$.

\begin{tabular}{|c|c|c|c|c|c|c|c|}
\hline PARCELAS & $T p$ & $T s$ & $E m$ & $\mathrm{CE}$ & $f c$ & $H(O-2)$ & $H(4-6)$ \\
\hline & $\min$ & $\min$ & $\mathrm{mm} \mathrm{h}^{-1}$ & $(\%)$ & $\mathrm{mm} \mathrm{h}^{-1}$ & $(\%)$ & $\%$ \\
\hline \multicolumn{8}{|l|}{ NORTE } \\
\hline BE01 & 3'02" & $3^{\prime} 48^{\prime \prime}$ & 0,32 & 0,62 & 54,65 & 3,21 & 6,32 \\
\hline $\mathrm{BE} 02$ & $7^{\prime} 02 "$ & 10'09" & 1,45 & 2,63 & 53,19 & 2,11 & 6,05 \\
\hline BE03 & 5' 24" & $10^{\prime} 36^{\prime \prime}$ & 0,16 & 0,29 & 54,89 & 4,47 & 5,14 \\
\hline BE04 & $5^{\prime} 24^{\prime}$ & $19^{\prime} 36^{\prime \prime}$ & 0,42 & 0,76 & 54,12 & 4,58 & 8,63 \\
\hline BE05 & 14'07" & $15^{\prime} 49^{\prime \prime}$ & 1,07 & 1,94 & 53,33 & 4,40 & 8,00 \\
\hline BE06 & 7' 25" & 9'00" & 0,17 & 0,31 & 54,78 & 2,69 & 4,88 \\
\hline BE07 & 6' $42 "$ & $7^{\prime} 07^{\prime \prime}$ & 3,49 & 6,34 & 49,35 & 2,87 & 4,70 \\
\hline BE08 & 2' 35" & $5^{\prime} 15^{\prime \prime}$ & 9,02 & 16,39 & 44,19 & 3,61 & 5,53 \\
\hline BE09 & 3' 26" & $3^{\prime} 56^{\prime \prime}$ & 3,38 & 6,15 & 51,36 & 5,28 & 10,7 \\
\hline \multirow[t]{2}{*}{ BE10 } & 12 ' $32 "$ & $15^{\prime} 00^{\prime \prime}$ & 0,47 & 0,87 & 54,36 & 2,21 & 3,05 \\
\hline & 6' 62" & 9' $86 "$ & 1,99 & 3,63 & 52,42 & 3,54 & 6,3 \\
\hline \multicolumn{8}{|l|}{ SUR } \\
\hline BE51 & 1'49" & 3'00" & 22,38 & 0,68 & 30,41 & 0,92 & 2,08 \\
\hline BE52 & 3' 26" & 9' $30^{\prime \prime}$ & 2,55 & 4,65 & 49,35 & 4,89 & 2,50 \\
\hline BE53 & $\mathrm{sp}$ & se & 0 & 0 & 55 & 1,13 & 2,07 \\
\hline BE54 & 1' 14" & 1'54" & 21,76 & 39,63 & 30,15 & 8,42 & 8,03 \\
\hline BE55 & 3'05" & $4^{\prime} 00 "$ & 1,55 & 0,28 & 49,53 & 2,76 & 5,06 \\
\hline BE56 & 1'23" & 1' 50 "' & 7,10 & 12,91 & 39,56 & 0,49 & 1,04 \\
\hline BE57 & $s p$ & se & 0 & 0 & 54,72 & 5,96 & 3,11 \\
\hline BE58 & 3' 11" & $5^{\prime} 11 "$ & 16,46 & 29,92 & 35,69 & 2,33 & 5,85 \\
\hline BE59 & 2' 56" & $6^{\prime} 00^{\prime \prime}$ & 16,96 & 30,82 & 35,67 & 1,09 & 2,54 \\
\hline \multirow[t]{2}{*}{$\mathrm{BE} 60$} & $5^{\prime} 32^{\prime \prime}$ & $6^{\prime} 00^{\prime \prime}$ & 1,80 & 3,27 & 52,92 & 2,46 & 3,01 \\
\hline & $2^{\prime} 50^{\prime \prime}$ & $4 ' 36 "$ & 9,06 & 16,22 & 43,33 & 3,05 & 3,53 \\
\hline
\end{tabular}

Tp, Tiempo de encharcamiento; Ts, Tiempo de escorrentía en salida; Em, Escorrentía media; CE, Coeficiente de escorrentía y fc, Tasa de infiltración final estable. Sin encharcamiento o encharcamiento insignificante (sp). Sin escorrentía (se) 


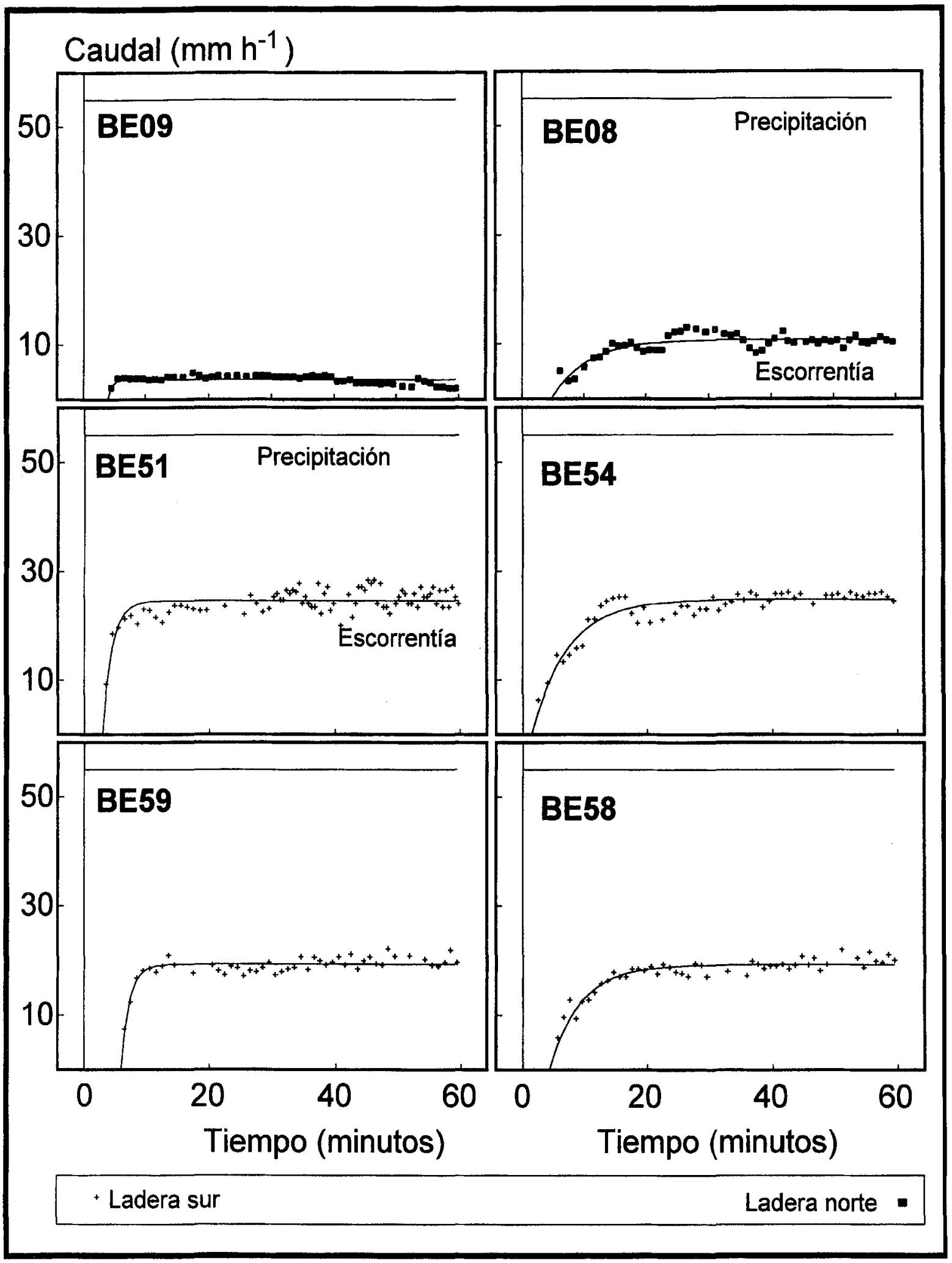

Figura 6. Hidrogramas de los seis experimentos con mayores tasas de escorrentía. En los restantes, catorce, la escorrentía fue insignificante. 
INFLUENCIA DE LA EXPOSICIÓN SOBRE LA PRODUCCIÓN DE SEDIMENTOS Y ESCORRENTÍAS

\subsection{Respuesta erosiva}

Los procesos de erosión están directamente relacionados con la producción de escorrentía. Los suelos que producen mayores caudales son los que aportan más sedimentos. En concreto, durante los 60 minutos de duración del chaparrón se han exportado una media por parcela de 0,07 $\mathrm{g}$ en las Hierbas, 0,3 $\mathrm{g}$ en el Matorral, 0,88 g en los Enlosados y 2,55 g en los Encostrados. Esto se ve reflejado en tasas de erosión y concentraciones de sedimentos dispares según el tipo de superficie (tabla 4).

Las diferencias entre la ladera norte y la sur son evidentes. Suelos menos erosionables en la ladera norte $\left(0,43 \mathrm{~g} \mathrm{l}^{-1}\right)$ que en la ladera sur $\left(0,69 \mathrm{~g}^{-1}\right)$, con menor exportación de sedimentos $(0,19$ y $1,4 \mathrm{~g})$ y obviamente, tasas de erosión mucho mayores en la ladera sur $\left(6,65 \mathrm{~g} \mathrm{~m}^{2} \mathrm{~h}^{-1}\right)$ que en la norte $\left(0,88 \mathrm{~g} \mathrm{~m}^{2} \mathrm{~h}^{-1}\right)$.

Tabla 4. Pérdida del suelo.

\begin{tabular}{|cccc|}
\hline PARCELAS & $\begin{array}{c}C s \\
\mathrm{~g} \mathrm{l}^{-1}\end{array}$ & $\begin{array}{c}\text { Sy } \\
\mathrm{g}\end{array}$ & $\begin{array}{c}\text { Tr } \\
\mathrm{g} \mathrm{m}^{2} \mathbf{h}^{-1}\end{array}$ \\
\hline NORTE & & & \\
BE01 & 0,71 & 0,05 & 0,23 \\
BE02 & 0,93 & 0,29 & 1,35 \\
BE03 & 0,98 & 0,04 & 0,16 \\
BE04 & 0,20 & 0,02 & 0,08 \\
BE05 & 0,61 & 0,15 & 0,65 \\
BE06 & 0,00 & 0,00 & 0,00 \\
BE07 & 0,31 & 0,30 & 1,10 \\
BE08 & 0,58 & 1,07 & 5,27 \\
BE09 & 0,00 & 0,00 & 0,00 \\
BE10 & 0,00 & 0,00 & 0,00 \\
Media & 0,43 & 0,19 & 0,88 \\
SUR & & & \\
BE51 & 1,19 & 5,19 & 26,67 \\
BE52 & 1,45 & 0,86 & 3,69 \\
BE53 & 0,00 & 0,00 & 0,00 \\
BE54 & 0,69 & 3,24 & 15,01 \\
BE55 & 0,83 & 0,22 & 1,29 \\
BE56 & 0,29 & 0,40 & 2,04 \\
BE57 & 0,00 & 0,00 & 0,00 \\
BE58 & 0,20 & 0,69 & 3,27 \\
BE59 & 0,69 & 2,84 & 11,77 \\
BE60 & 1,52 & 0,59 & 2,74 \\
Media & 0,69 & 1,40 & 6,65 \\
\hline
\end{tabular}

Cs, concentración de sedimentos; Sy, Sedimentos totales y Tr, Tasa de erosión 


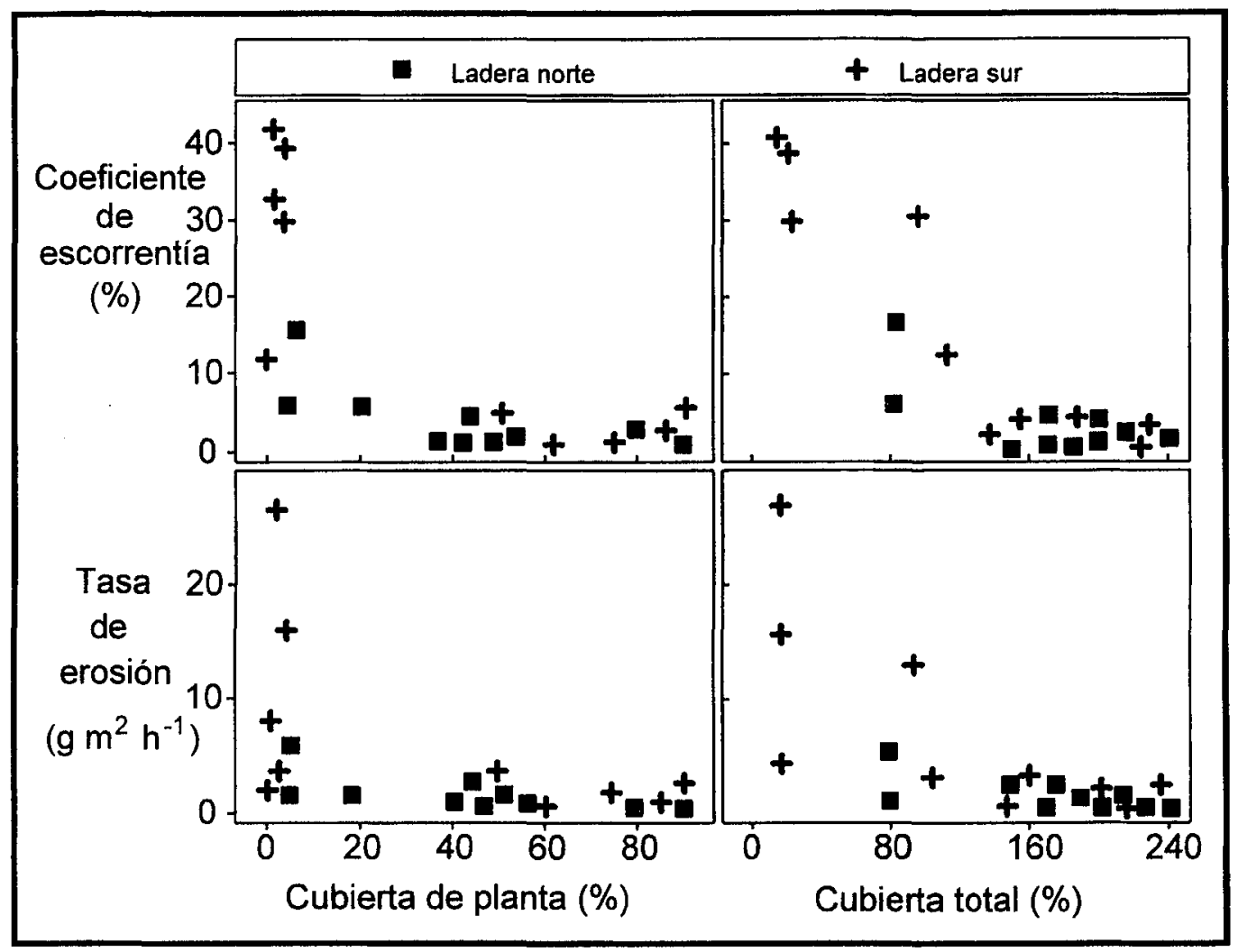

Figura 7. Relación de la cubierta de plantas y la cubierta total (plantas, \%; musgos, \%; líquenes, \%; bojarasca, \%; pedregosidad, \%; y altura de la vegetación, (m) con el coeficiente de escorrentía y la tasa de erosión.

\section{Discusión}

La influencia positiva de la vegetación en la reducción de las tasas de escorrentía y erosión vistas a lo largo de la exposición de los resultados ha sido tratada en otros trabajos (CERDÀ, 1995a; 1995b; CERDÀ et al., 1995; en prensa), confirmando estudios de otros autores (THORNES, 1980; SALA y CALVO, 1990; FRANCIS y THORNES, 1990a; WOO y LUK, 1990).

La vegetación es considerada el factor más importante en el control de la escorrentía y la erosión (WISCHMEIER, 1960; ELWELL y STOCKING, 1976; HUDSON, 1982; BERNDTSSON y LARSON, 1987). Es evidente que la relación entre la vegetación y la producción de sedimentos y escorrentía no es lineal, sino que responde a umbrales o bien es exponencial (ELWELL y STOCKING, 1976; LEE y SKOGERBOE, 1985; FRANCIS y THORNES, 1990b; CALVO et al., 1992). La influencia positiva de la vegetación y de la pedregosidad reside en la protección del suelo sobre los impactos de gota y en la mejora de las propiedades físicas, químicas y biológicas del suelo (porosidad, materia orgánica, etc.) (THORNES, 1976; MORGAN, 1986; POESEN e INGELMO-SÁNCHEZ, 1992). 
Este trabajo confirma las anteriores ideas (Fig. 7). Además de la influencia de la vegetación sobre los procesos de erosión y generación de arroyada superficial, estos últimos procesos también tienen claros efectos sobre la distribución de la vegetación. Es evidente que una visión global debe ser aplicada para entender el efecto de la vegetación sobre los procesos geomorfológicos, así como las interrelaciones entre factores y las interacciones entre procesos (THORNES, 1990; LÓPEZ BERMÚDEZ et al., 1992).

La dinámica de la vegetación en los ecosistemas semiáridos favorece su distribución en manchas y zonas desnudas entre-matas, lo que en parte es debido a la competencia por el agua (THORNES, 1985), pero también a la dinámica erosiva. Esta última genera espacios desnudos productores de sedimentos y espacios vegetados en los que agua y sedimentos se acumulan durante los eventos más intensos. La distribución en manchas de la vegetación es una de las características más destacable de la ladera sur estudiada, donde el esparto (Stipa tenacissima L.) domina la estructura de la comunidad vegetal, situación muy extendida en el sudeste peninsular. Esto también determina que los procesos dependientes de la vegetación: intercepción, infiltración, escorrentía y erosión sean muy variables espacialmente, cómo también ocurre en otros ambientes semiáridos y de montaña (SCOGING, 1982; IMESON, 1984; RUIZ FLAÑO et al., 1991; RUIZ FLAÑO, 1993; CERDÀ, 1995a).

A escala de ladera es evidente la influencia de la vegetación y su distribución en la dinámica de los procesos geomorfológicos superficiales, y al revés (SÁNCHEZ y PUIGDEFÁBREGAS, 1994a; 1994b), a escala de cuenca es claro el control ejercido por la exposición. De los resultados aquí expuestos se desprende que las laderas norte generan menores escorrentías, éstas contienen menores cargas sólidas, se inician más tarde y dan lugar a tasas de erosión mucho menores que las laderas sur. Otra diferencia es que la arroyada es mucho más contrastada dentro de la ladera sur, debido a la estructura de la vegetación en un mosaico de macollas de esparto y zonas de pasillos desnudas, que en la ladera norte donde la cubierta vegetal es más homogénea (Fig. 7).

Estudios en los que se incluyan ambas zonas, desnudas y vegetadas, bien mediante experimentos controlados, bien mediante parcelas convencionales, serán necesarios para conocer el efecto del cambio de escala sobre los procesos de generación de escorrentía y erosión de las laderas. En el desierto del Negev, con una cubierta vegetal mucho menor y suelos muy poco desarrollados se ha demostrada la discontinuidad de la escorrentía en parcelas mayores (YAIR y LAVEE, 1985), lo que hace muy probable que esto también suceda en zonas con vegetación distribuida en matas como la aquí estudiada.

A pesar de la influencia de la exposición, la elevada variabilidad espacial en los ambientes semiáridos hace que en la ladera sur existan suelos con escorren- 
ARTEMI CERDÀ

tías y tasas de erosión muy bajas, correspondientes a suelos vegetados. Pero también existen suelos con arroyadas muy activas en las zonas desnudas, las cuales son más abundantes en la ladera sur (Fig. 7), lo que genera mayores tasas medias de erosión y escorrentía.

Esta mayor heterogeneidad de la vegetación, y con ello de los procesos geomórficos, en la ladera sur puede ser entendida cómo una estrategia para generar infiltraciones mayores y más profundas en la zona vegetada a partir de la escorrentía generada en las zonas desnudas. Esto mismo sucede con los sedimentos que se acumulan en las partes altas de las macollas, y que facilitan el crecimiento de las matas por el aporte de nuevos nutrientes (SÁNCHEZ, 1995).

Estudios más detallados sobre la ecología de Stipa tenacissima L. y de su interrelación con los procesos geomórficos son necesarios para conocer el comportamiento de estos ecosistemas de forma global. Además, las nuevas aproximaciones al tema de la erosión deben realizarse desde distintas escalas de observación, con una visión jerárquica de los procesos geomorfológicos y su interrelación con los factores bióticos y abióticos.

En el caso que nos ocupa, además de la aproximación a escala de matas, estudios del comportamiento global de las laderas o partes de las laderas en las que se incluyan zonas desnudas y vegetadas son necesarios. Posiblemente, la infiltración de la escorrentía y la deposición de sedimentos en las matas favorezca tasas de erosión y escorrentía muy bajas a nivel de ladera. Además, las pérdidas por transmisión (WALLING, 1983) dentro de las laderas pueden ser esenciales para explicar la dinámica geomorfológica de las vertientes en ambientes semiáridos.

Tanto la escorrentía como la erosión están claramente influenciadas por la exposición. La ladera norte mantiene mayores tasas de infiltración, la escorrentía es retrasada y escasa, y la pérdida de suelo es insignificante en relación con la ladera sur. La distribución de la vegetación en un mosaico de macollas de esparto y zonas desnudas da lugar a una elevada variabilidad de los procesos de escorrentía y erosión, especialmente en la ladera más árida, la sur. Las relaciones entre la vegetación y la arroyada no son lineales y presentan umbrales muy marcados. La elevada variabilidad espacial de los procesos geomorfológicos hace difícil su modelización y predicción.

\section{Agradecimientos}

Este estudio ha sido realizado dentro del proyecto Modelling and exploring the impact of climate change on Ecosystem degradation, bydrology and land use along a transect across the Mediterranean (ERMES) financiado por la Unión Europea a través del programa Climatology and Natural Hazards (EV5VCT91-0023). A. Cerdà agradece al Ministerio de Educación y Ciencia la concesión de una beca postdoctoral en el extranjero que le ha permitido participar en este proyecto y a Adolfo Calvo, Amparo Soriano y Carolina Boix la colaboración en el trabajo de campo. 
INFLUENCIA DE LA EXPOSICIÓN SOBRE LA PRODUCCIÓN DE SEDIMENTOS Y ESCORRENTÍAAS

\section{Referencias bibliográficas}

ABRAHAMS, A.D. y PARSONS, A.J. (1994): Geomorphology of Desert environments. Chapman and Hall, Cambridge, $674 \mathrm{p}$.

BERNDTSSON, R. y LARSON, M. (1987): Spatial variability of infiltration in a semiarid environment. Journal of bydrology, 90, 117-133.

BOIX, C., CALVO, A., SORIANO, M.D., y TIENESSEN, I. (1994): Variabilidad espacio-temporal de la agregación de suelos en laderas mediterráneas. En J. ARNÁEZ, J.M. GARCÍA RUIZ y A. GÓMEZ VILLAR, (Eds.,): Geomorfología en España, 289-302.

CALVO, A., HARVEY, A. y PAYÀ, J. (1992): Response of badland surfaces in south east Spain to simulated rainfall. Cuaternario y Geomorfología, 6, 3-14.

CALVO, A., SORIANO, M.D., BOIX, C., CERDÀ, A., TIEMESSEN, I. y IMESON, A.C. (1994): Report of the Alicante Study Areas. Proyecto de Invertigación ERMES. $168 \mathrm{ff}$.

CERDÀ, A. (1993): La Infiltración en los Suelos del País Valenciano. Factores $y$ Variaciones Espacio-Temporales. Tesis Doctoral Inédita. Universitat de València. $357 \mathrm{p}$.

CERDÀ, A. (1995a): Factores y Variaciones Espacio-Temporales de la Infiltración en los Ecosistemas Mediterráneos. Editorial Geoforma. Logroño, $151 \mathrm{p}$.

CERDÀ, A. (1995b): Seasonal variability of infiltration rates under contrasting slope conditions in Southeast Spain. Geoderma.

CERDÀ, A., CALVO, A., SORIANO, M.D., BOIX, C. y IMESON, A. (1995): Escorrentía y movilización de suelo en ambiente semiárido. Influencia de la cubierta vegetal. En Actas del XX Reunión Nacional de Suelos: Degradación y conservación de suelos en áreas de montaña, 109-117.

CERDÀ, A., CALVO, A., SORIANO, M.D., BOIX, C. y IMESON, A. (en prensa): Influencia de la vegetación en la dinámica erosiva de los suelos en un ambiente semiárido. El ejemplo de Stipa tenacissima L. En Actas del VII Colóquio Ibérico de Geografía. Cáceres, 25-30 de Septiembre de 1995.

CHURCHILL, R.R. (1981): Aspect-related differences in Badlands slope morphology. Annals of the Asociation of American Geographers, 71 (3) 374-388

CHURCHILL, R.R. (1982): Aspect-induced differences in hillslopes processes. Earth Surface Processes and Landforms, 7, 171-182.

COOKE, R.U., WARREN, A. y GOUDIE, A.S. (1993): Desert Geomorphology. UCL Press, London.

ELÍAS CASTILLO, J. y RUIZ BELTRAN, L. (1977): Precipitaciones máximas en España. Ministerio de Agricultura, Madrid, 545 p. 
ARTEMI CERDÀ

ELWELL, H.A. y STOCKING, M.A. (1976): Vegetal cover to estimate soil erosion hazard in Rodhesia. Geoderma, 15, 61-70.

FRANCIS, C.F. y THORNES, J.B. (1990a). Matorral: Erosion and Reclamation. En ALBADALEJO, J., STOCKING, M.A. y DÍAZ, E. (Eds.): Degradacion $y$ Regeneración del suelo en condiciones ambientales mediterráneas, 86-115.

FRANCIS, C.F. y THORNES, J.B. (1990b). Runoff hydrographs from Three Mediterranean Vegetation Cover Types. En J.B. THORNES (Ed.): Vegetation and Erosion, 365-384.

GONZÁLEZ HIDALGO, J.C. (1992): Pautas Espaciales de la Erosión Hídrica en el Semiárido Aragonés. Exposición Topográfica y Cubierta Vegetal, Factores de Erosión. Tesis Doctoral Inédita, Universidad de Zaragoza, 375 p.

GONZÁLEZ HIDALGO, J.C. (1994): Relaciones hídricas suelo/planta afectadas por la exposición de la ladera. Studia OEcologica, X-XI, 89-100.

GREGORY, K.J. y WALLING, D.E. (1973): Drainage Basins Forms and Processes: A Geomorphological Approach. Arnold, London, 458 p.

HUDSON, N. (1982): Conservación de suelo. Reverté, s. a. Barcelona, 335 p.

IMESON, A.C. (1984): An eco-geomorphological approach to the soil degradation and erosion problem. En FANTECHI, R. y MARGARIS, N.S. (Eds.): Desertification in Europe, 110-25.

KIRKBY, M.J. (1986): Conditions for valley asymetry derived from a slope evolution model. Working paper 477, Leeds Univ., School of Geography.

LEE, C.R. y SKOGERBOE, J.G. (1985): Quantification of erosion control by vegetation on problem soils. En EL SWAIFY, W.C., MOLDENHAUER, W.C. y LO, A. (Eds.): Soil Erosion and Conservation. Soil Conservation Soc. America, 437-444.

LÓPEZ BERMÚDEZ, F., RUBIO RECIO, J.M. y CUADRAT, J.M. (1992): Geografía Física. Editorial Cátedra, 594 p.

MARGALEF, R. (1986): La Ecología. Barcelona, Omega.

MORGAN, R.P.C. (1986): Soil Erosion and Conservation. Longman, New York, $298 \mathrm{p}$.

POESEN, J. e INGELMO SÁNCHEZ, F. (1992). Runoff and sediment yields from topsoils with different porosity as affected by rock fragment cover and position. Catena, 19. 451-474.

RUIZ FLAÑO, P. (1993): Procesos de erosión en campos abandonados del Pirineo. Geoforma Ediciones. Logroño, 191 p.

RUIZ FLAÑO, P., GARCÍA RUIZ, J.M., ORTIGOSA IZQUIERDO, L.M. y LASANTA MARTÍNEZ, T. (1991): La producción de sedimentos en suspensión en microambientes geomorfológicos de campos abandonados. En Actas del XII Congreso Nacional de Geografía, Valencia, 69-76. 
INFLUENCIA DE LA EXPOSICIÓN SOBRE LA PRODUCCIÓN DE SEDIMENTOS Y ESCORRENTÍAS

SALA, M. y CALVO, A. (1990): Response of four different mediterranean vegetation types to runoff and erosion. En THORNES, J.B. (Ed.): Vegetation and Erosion: Process and Environments, 347-62.

SÁNCHEZ, G. y PUIGDEFÁBREGAS, J. (1994a): Interactions of plant growth and sediment movement on slopes in a semi-arid environment. Geomorpbology, 9, 243-260.

SÁNCHEZ, G. y PUIGDEFÁBREGAS, J. (1994b): Simulación del desarrollo de macollas de esparto (Stipa tenacissima $L$.) en relación a la arquitectura y el transporte de sedimentos. Studia Oecologica, X-XI, 211-219.

SÁNCHEZ, G. (1995): Arquitectura y dinámica de las matas de esparto (Stipa tenacissima L.), efectos en el medio e interacciones con la erosión. Tesis Doctoral. Universidad Autónoma de Madrid, 119 p.

SCOGING, H. (1982): Spatial variations in infiltration runoff and erosion on hillslopes in semi-arid Spain. En BRYAN, R. y YAIR, A. (Eds.): Badland geomorpbology and piping, 89-112.

SORIANO, M.A., CERDÀ, A., BOIX, C., CALVO, A. y IMESON, A.C: (1995): Características de los suelos en ambientes semiáridos. Estabilidad y distribución de los agregados. Alicante. En Actas del XX Congreso Nacional de Suelos: Degradación y conservación de suelos en áreas de montaña, 285-292.

THORNES, J.B. (1976): Semi-arid erosion systems: case studies from Spain". Geogr, Papers, $\mathrm{n}^{\circ}$ 7, London School of Economics.

THORNES, J.B. (1980): Erosional processes of running water and their spatial and temporal controls: a theoretical viewpoint. En KIRKBY, M.J. y MORGAN, R.P.C. (Eds.): Soil Erosion, 129-182.

THORNES, J.B. (1985): The ecology of erosion. Geography, 70 (3), 222-36.

THORNES, J.B. (1990): The Interaction of Erosional and Vegetational Dynamics in Land Degradation: Spatial Outcomes. En THORNES, J.B. (Ed.): Vegetation and erosion: Processes and Environments,41-54.

WALLING, D.E. (1983): The sediment delivery problem. Journal of Hydrology, $65,209-237$.

WEAVER, A. van B. (1991): The distribution of soil erosion as a function of slope aspect and parent material in Ciskei, Southern Africa. Geojournal, 23 (1) 29-34.

WISCHMEIER, V.H. (1960): Cropping-management factor evaluations for a universal soil-loss equation. Proc. Soil Sci. Soc. Am., 24, 322-326

WOO, M.-K. y LUK, S.-H. (1990): Vegetation effects on soil and water losses on weathered granitic hillslopes, south China. Pbysical Geograpby, 11 (1) 1-16.

YAIR, A. y LAVEE, H. (1985): Runoff generation in arid and semi-arid zones. En ANDERSON y BURT, T.P. (Eds.): Hydrological Forecasting, 183-220. 